\title{
Manifestly scale-invariant regularization and quantum effective operators
}

\author{
D. M. Ghilencea* \\ Theoretical Physics Department, National Institute of Physics and Nuclear Engineering (IFIN-HH), \\ Bucharest 077125, Romania \\ Theory Division, CERN, 1211 Geneva 23, Switzerland
}

(Received 3 March 2016; published 4 May 2016)

\begin{abstract}
Scale-invariant theories are often used to address the hierarchy problem. However the regularization of their quantum corrections introduces a dimensionful coupling (dimensional regularization) or scale (PauliVillars, etc) which breaks this symmetry explicitly. We show how to avoid this problem and study the implications of a manifestly scale-invariant regularization in (classical) scale-invariant theories. We use a dilaton-dependent subtraction function $\mu(\sigma)$ which, after spontaneous breaking of the scale symmetry, generates the usual dimensional regularization subtraction scale $\mu(\langle\sigma\rangle)$. One consequence is that "evanescent" interactions generated by scale invariance of the action in $d=4-2 \epsilon$ (but vanishing in $d=4$ ) give rise to new, finite quantum corrections. We find a (finite) correction $\Delta U(\phi, \sigma)$ to the one-loop scalar potential for $\phi$ and $\sigma$, beyond the Coleman-Weinberg term. $\Delta U$ is due to an evanescent correction $(\propto \epsilon)$ to the field-dependent masses (of the states in the loop) which multiplies the pole $(\propto 1 / \epsilon)$ of the momentum integral to give a finite quantum result. $\Delta U$ contains a nonpolynomial operator $\sim \phi^{6} / \sigma^{2}$ of known coefficient and is independent of the subtraction dimensionless parameter. A more general $\mu(\phi, \sigma)$ is ruled out since, in their classical decoupling limit, the visible sector (of the Higgs $\phi$ ) and hidden sector (dilaton $\sigma$ ) still interact at the quantum level; thus, the subtraction function must depend on the dilaton only, $\mu \sim \sigma$. The method is useful in models where preserving scale symmetry at quantum level is important.
\end{abstract}

DOI: 10.1103/PhysRevD.93.105006

\section{INTRODUCTION}

There has recently been a renewed interest in the scale invariance symmetry to address the hierarchy or the cosmological constant problems. Scale symmetry is not a symmetry of the real world since it requires that no dimensionful parameters be present in the Lagrangian. One can impose this symmetry on the Lagrangian at the classical level, to forbid any mass scales. At the quantum level, the anomalous breaking of scale symmetry is, in general, expected. This is because regularization of the loop corrections breaks this symmetry explicitly, either by introducing a dimensionful coupling as in dimensional regularization (DR) or a mass scale (Pauli-Villars, cutoff regularizations, etc). Therefore, the presence of a subtraction (or renormalization) scale $\mu$ breaks explicitly the (classical) scale invariance of the theory and ruins the symmetry one actually wants to study. In DR the scale $\mu$ relates the dimensionless couplings to the dimensionful ones, once the theory is continued analytically from $d=4$ to $d=4-2 \epsilon$ dimensions. For example, the quartic coupling $\left(\lambda_{\phi}\right)$ of a Higgs-like scalar field $\phi$ acquires a mass dimension, since

*dumitru.ghilencea@cern.ch

Published by the American Physical Society under the terms of the Creative Commons Attribution 3.0 License. Further distribution of this work must maintain attribution to the author(s) and the published article's title, journal citation, and DOI.

$$
\lambda_{\phi}=\mu^{2 \epsilon}\left(\lambda_{\phi}^{(r)}+\sum_{n} a_{n} / \epsilon^{n}\right)
$$

where renormalized $\lambda_{\phi}^{(r)}$ is dimensionless. Thus, the DR scale $\mu$ breaks scale invariance. ${ }^{1}$

To avoid this problem in theories in which scale invariance must be preserved during regularization, we use a scheme in which the couplings become field dependent, something familiar in string theory. Indeed, one can replace the scale $\mu$ by a function $\mu(\sigma), \mu \rightarrow \mu(\sigma)$ $[1,2]$ where the field $\sigma$ is the dilaton ${ }^{2}$; for example, $\mu(\sigma) \propto \sigma$. Of course, $\sigma$ must subsequently acquire a nonzero (finite) vacuum expectation value (vev); otherwise, this relation does not make sense due to vanishing power $(\epsilon \rightarrow 0)$ in Eq. (1). Also, one cannot just replace $\mu$ by the vev of the field $\sigma$, since this would simply bring back the original problem. One therefore needs a spontaneous breaking of the scale symmetry. When the (dynamical) field $\sigma$ acquires a nonzero vev, scale invariance is broken with the dilaton $\sigma$ as its Goldstone mode. This can happen in a framework which includes (conformal) gravity in which the dilaton vev is related to the Planck scale. In this paper we shall not include gravity, but assume the

\footnotetext{
${ }^{1}$ The exact $S$ matrix is renormalization scale independent. But in perturbation theory we truncate the series, so there is always a residual renormalization scheme dependence, which must be minimized.

${ }^{2}$ We also consider a more general dependence $\mu=\mu(\phi, \sigma)$ where $\phi$ is our scalar (Higgs-like) field.
} 
dilaton acquires a vev spontaneously (fixed, e.g., by Planck scale physics) and search for solutions $\langle\sigma\rangle \neq 0$.

The goal of this paper is to investigate the quantum implications of a manifestly scale-invariant regularization of a theory that is classically scale invariant, using a dilatondependent subtraction "scale." This is important since scale-invariant theories, see, e.g., [3-22], do not seem to be renormalizable $[23,24]$, in which case the regularization of the loops should preserve all initial symmetries to avoid regularization artifacts [25]. This motivated our work and is relevant for theories which study scale invariance at the quantum level.

This paper continues a previous study [2], with notable differences and new results that we outline below. Consider a scale-invariant theory of Higgs-like $\phi$ and dilaton $\sigma$ (other fields may be present). In "usual" DR, quartic couplings become dimensionless by replacing $\lambda \rightarrow \mu^{2 \epsilon} \lambda$ [see Eq. (1)] and this changes also the scalar potential $V(\phi, \sigma)$. For a field-dependent subtraction function $\mu(\sigma)$, this change is $V(\phi, \sigma) \rightarrow \tilde{V} \equiv \mu^{2 \epsilon}(\sigma) V(\phi, \sigma)$, which is scale invariant in $d=4-2 \epsilon$ (as it should). So $\tilde{V}$ acquired new "evanescent" interactions $^{3}$ due to the field dependence of $\mu(\sigma)$. This step generates new, finite corrections at the quantum level.

For example, we obtain a scale-invariant one-loop potential $U(\phi, \sigma)$ which contains a finite (quantum) correction $\Delta U(\phi, \sigma)$ beyond the usual Coleman-Weinberg (CW) term [26,27] for the Higgs $\phi$ and dilaton $\sigma . \Delta U$ is a new correction overlooked by previous studies [2] and at the technical level it arises when the evanescent correction $(\propto \epsilon)$ to the field-dependent masses ${ }^{4}$ in the loop multiplies the poles $1 / \epsilon$ of the loop integrals, thus giving a finite contribution. Note that $\Delta U$ contains nonpolynomial operators like $\phi^{6} / \sigma^{2}$ of the known coefficient; such new operators generate, in turn, polynomial effective operators when expanded about $\langle\sigma\rangle \neq 0(\langle\sigma\rangle$ can be arranged to be much larger than the electroweak vev $\langle\phi\rangle$, see later).

The subtraction function cannot also depend on the Higgs field $\phi$ (as in [2]) since this would bring the nondecoupling quantum effects of the visible sector $(\phi)$ to the hidden sector $(\sigma)$ even in their classical decoupling limit. As a result, we have a dilaton-only dependent subtraction function which must be of the form $\mu(\sigma)=$ $z \sigma$ where $z$ is an arbitrary dimensionless constant. Unlike total $U(\phi, \sigma), \Delta U$ is independent of the subtraction scale $(z\langle\sigma\rangle)$, being finite. Of course physics must be independent of $z$ (and thus of $z\langle\sigma\rangle$ ), so we check that our full potential does respect the Callan-Symanzik equation in our approach; see [5] for a discussion.

Assuming the couplings are initially tuned at the classical level to enforce a hierarchy $\langle\phi\rangle \ll\langle\sigma\rangle$, we show

\footnotetext{
${ }^{3}$ These are defined as interactions absent in $d=4(\epsilon=0)$ but generated in $d=4-2 \epsilon$ by scale invariance.

${ }^{4}$ These masses are $\partial^{2} \tilde{V} / \partial \alpha \partial \beta, \alpha, \beta=\phi, \sigma$ and contain corrections $\alpha \epsilon$ from derivatives of $\mu(\sigma)$; see later.
}

the quantum correction to the mass of $\phi$ that is due to $\Delta U$ remains small without additional tuning of the couplings. Using this symmetry-preserving regularization and spontaneous breaking of scale symmetry, one can address the hierarchy problem at higher loops.

In the case of a field-dependent subtraction function there is no initial subtraction scale present in the theory, so there is no dilatation anomaly. Note that it is possible that a theory be quantum scale invariant and the couplings still run with the momentum scale $[5,6]$. One first performs loop calculations with a field-dependent regularization. After spontaneous breaking of scale symmetry $\langle\sigma\rangle \neq 0$, the subtraction scale and all masses and vevs of the theory are generated, proportional to ${ }^{5}\langle\sigma\rangle$. After regularization and renormalization one can eventually decouple the dilaton by taking the limit of vanishing couplings for it, while keeping the masses of the theory fixed [5]. ${ }^{6}$

After introducing the model (Sec. II) we present the scale-invariant result of the one-loop potential for a general subtraction function (Sec. III); this function is shown to depend on the dilaton only $\mu=z \sigma$ (Sec. IV). The implications for the mass of $\phi$ are addressed (Sec. V) with Callan-Symanzik equation verified in Sec. VI, followed by Conclusions.

\section{A GENERIC MODEL}

Consider a Lagrangian with two real scalar fields

$$
\mathcal{L}=\frac{1}{2} \partial_{\mu} \phi \partial^{\mu} \phi+\frac{1}{2} \partial_{\mu} \sigma \partial^{\mu} \sigma-V(\phi, \sigma) .
$$

The potential in $d=4$ scale-invariant theories has the structure $V(\phi, \sigma)=\sigma^{4} W(\phi / \sigma)$ and is an homogeneous function; therefore, it satisfies the relation $^{7}$ $\phi \partial V / \partial \phi+\sigma \partial V / \partial \sigma=4 V$. Using this and with the notation $x=\phi / \sigma$, the extremum conditions for $V$ $\left(\partial_{\phi} V=\partial_{\sigma} V=0\right)$ can be written as $W(x)=W^{\prime}(x)=0$ if we assume that $\langle\sigma\rangle,\langle\phi\rangle \neq 0$. One of these conditions fixes the ratio of the fields vevs, while the second implies a relation (tuning) among the couplings of the theory. If $x_{0}=$ $\langle\phi\rangle /\langle\sigma\rangle$ is a solution to these two conditions, then $\langle\phi\rangle$ is proportional to $\langle\sigma\rangle$ which means that a flat direction exists [3] in this theory, along the line in the plane $(\phi, \sigma)$ with $\phi / \sigma=x_{0}$. Also since $W\left(x_{0}\right)=0$ on the ground state, then $V(\langle\phi\rangle,\langle\sigma\rangle)=0$. Thus, in theories with spontaneous breaking of scale symmetry, a vanishing cosmological constant at a given order of perturbation theory demands a tuning of the relation among couplings, in that order.

\footnotetext{
${ }^{5}$ In a scale-invariant setup, in the absence of gravity, one can only predict ratios of fields' vevs.

${ }^{6}$ With scale invariance broken by gravity, the dilaton couplings to matter are expected to be very small.

${ }^{7}$ To find this relation, use that $V(\alpha \phi, \alpha \sigma)=\alpha^{4} V(\phi, \sigma)$ (homogeneous), differentiate with respect to $\alpha$, and set $\alpha=1$.
} 
An example of a scale-invariant potential that we consider below is

$$
V=\frac{\lambda_{\phi}}{4} \phi^{4}+\frac{\lambda_{m}}{2} \phi^{2} \sigma^{2}+\frac{\lambda_{\sigma}}{4} \sigma^{4}
$$

where note that the couplings can depend on $\phi / \sigma$, that more fields be present, etc.

In the simple case the couplings are independent of $\phi / \sigma$; minimizing this $V$ gives

$$
\begin{aligned}
& \langle\phi\rangle\left(\lambda_{\phi}\langle\phi\rangle^{2}+\lambda_{m}\langle\sigma\rangle^{2}\right)=0, \\
& \langle\sigma\rangle\left(\lambda_{m}\langle\phi\rangle^{2}+\lambda_{\sigma}\langle\sigma\rangle^{2}\right)=0 .
\end{aligned}
$$

One can distinguish the following situations:

Case (a): The ground state is $\langle\sigma\rangle=0,\langle\phi\rangle=0$ and both fields are massless.

Case (b): A more interesting case that we study in this paper is that of spontaneous breaking of the scale symmetry when $\langle\sigma\rangle \neq 0$. A solution to both equations in (4) then exists for $\langle\sigma\rangle<\infty$ (finite). Then also $\langle\phi\rangle \neq 0$, and a nontrivial ground state exists provided that $\lambda_{m}^{2}=\lambda_{\phi} \lambda_{\sigma}$ and $\lambda_{m}<0$, which we assume to be true in the following. Then

$$
\begin{aligned}
& \frac{\langle\phi\rangle^{2}}{\langle\sigma\rangle^{2}}=-\frac{\lambda_{m}}{\lambda_{\phi}}, \Rightarrow V=\frac{\lambda_{\phi}}{4}\left(\phi^{2}+\frac{\lambda_{m}}{\lambda_{\phi}} \sigma^{2}\right)^{2}, \\
& \left(\lambda_{m}^{2}=\lambda_{\sigma} \lambda_{\phi} ; \lambda_{m}<0\right) .
\end{aligned}
$$

Then a spontaneous breaking of the scale symmetry implies electroweak symmetry breaking at tree level, with a vanishing cosmological constant; it also demands the existence of a finite (nonzero) scale $\langle\sigma\rangle$ (unknown) in the theory. All scales are then generated by $\langle\sigma\rangle{ }^{8}$.

Further, one shifts the fields $\phi \rightarrow \phi+\langle\phi\rangle$ and $\sigma \rightarrow$ $\sigma+\langle\sigma\rangle$ and Taylor expands about the ground state. The mass eigenstates are $\tilde{\phi}=\phi \cos \alpha+\sigma \sin \alpha$ and $\tilde{\sigma}=$ $-\phi \sin \alpha+\sigma \cos \alpha$ where $\tan ^{2} \alpha=-\lambda_{m} / \lambda_{\phi}>0$. A flat direction exists, so one field (dilaton $\sigma$ ) is massless while the second field $\phi$, which would be the Higgs boson in a realistic model, has a mass

$m_{\tilde{\phi}}^{2}=2 \lambda_{\phi}\left(1-\lambda_{m} / \lambda_{\phi}\right)\langle\phi\rangle^{2}=-2 \lambda_{m}\left(1-\lambda_{m} / \lambda_{\phi}\right)\langle\sigma\rangle^{2}$.

Ultimately, scale invariance is expected to be broken by Planck physics; thus, $\sigma$ will acquire a large vev, $\langle\sigma\rangle \sim M_{\text {Planck }}$. If one would like to implement a hierarchy with $m_{\tilde{\phi}} \sim\langle\phi\rangle \sim \mathcal{O}(100 \mathrm{GeV}) \ll\langle\sigma\rangle$, one should tune accordingly the couplings $\lambda_{\sigma} \ll\left|\lambda_{m}\right| \ll \lambda_{\phi}$. Such a

\footnotetext{
${ }^{8}$ Values $\langle s\rangle=0$ and $\langle s\rangle=\infty$, with $s=\phi, \sigma$ are excluded, unless Eq. (4) is implemented in the sense of a limit (also the couplings $\lambda_{m, \phi, \sigma}$ can depend on the ratio of the two fields but such case requires assumptions about this dependence, not studied here). Thus, although there are no scales in the initial theory, infinite or vanishing values for the fields are excluded in spontaneous breaking of scale symmetry.
}

hierarchy of couplings is possible $[15,28]$. It was observed [15] that the shift symmetry of the dilaton enables the couplings $\lambda_{m, \sigma}$ to remain ultraweak under renormalization group evolution.

One would like to know if at the quantum level this treelevel tuning is enough or if additional tuning (beyond that of $\lambda_{m}$ ) is required to maintain this hierarchy and $m_{\tilde{\phi}}$ light. Indeed, at one-loop dangerous corrections can emerge, such $m_{\tilde{\phi}}^{2} \sim \lambda_{\phi}^{2}\langle\sigma\rangle^{2}$, that would require additional tuning (of $\lambda_{\phi}$ ) and would reintroduce the hierarchy problem.

\section{SCALE INVARIANCE OF ONE-LOOP POTENTIAL AND EFFECTIVE OPERATORS}

To compute the one-loop potential, consider the DR scheme in $d=4-2 \epsilon$. Then, the mass dimensions are $[L]=d,[\phi]=[\sigma]=(d-2) / 2$; the couplings $\lambda_{\phi}, \lambda_{m}, \lambda_{\sigma}$ are dimensionful, $[\lambda]=\left[V^{(4)}\right]=4-d$. To render the couplings dimensionless, one uses the DR scale $\mu$ and replaces $\lambda \rightarrow \lambda \mu^{4-d}$. The scale $\mu$ breaks the classical scale invariance. To avoid this problem and to preserve this symmetry during regularization, replace $\mu$ by a field-dependent function (unknown ${ }^{9}$ ) so $\mu^{4-d} \rightarrow \mu(\phi, \sigma)^{4-d}$. Then the actual Lagrangian is

$$
\begin{aligned}
L & =\frac{1}{2} \partial_{\mu} \phi \partial^{\mu} \phi+\frac{1}{2} \partial_{\mu} \sigma \partial^{\mu} \sigma-\tilde{V}(\phi, \sigma), \\
\tilde{V}(\phi, \sigma) & \equiv \mu(\phi, \sigma)^{4-d} V(\phi, \sigma),
\end{aligned}
$$

$L$ is scale invariant in $d$ dimensions, $[\tilde{V}]=d,[V]=2 d-4$. Denote by $\tilde{M}^{2}$ the field-dependent mass matrix

$$
\left(\tilde{M}^{2}\right)_{\alpha \beta}=\frac{\partial^{2} \tilde{V}(\phi, \sigma)}{\partial \alpha \partial \beta}, \quad \alpha, \beta=\phi, \sigma .
$$

Then the one-loop potential, which manifestly respects scale invariance, is found from ${ }^{10}$

$$
\begin{aligned}
U & =\tilde{V}(\phi, \sigma)-\frac{i}{2} \int \frac{d^{d} p}{(2 \pi)^{d}} \operatorname{Tr} \ln \left[p^{2}-\tilde{M}^{2}(\phi, \sigma)+i \varepsilon\right] \\
& =\tilde{V}(\phi, \sigma)-\frac{1}{2} \frac{1}{(2 \pi)^{d}} \Gamma[-d / 2] \operatorname{Tr}\left[\pi \tilde{M}^{2}(\phi, \sigma)\right]^{d / 2} \\
& =\tilde{V}(\phi, \sigma)-\frac{1}{64 \pi^{2}} \sum_{s=\phi, \sigma} \tilde{M}_{s}^{4}\left[\frac{2}{4-d}+\ln \kappa-\ln \tilde{M}_{s}^{2}\right], \\
\kappa & \equiv 4 \pi e^{3 / 2-\gamma_{E}} .
\end{aligned}
$$

\footnotetext{
${ }^{9}$ This function is assumed to be nonzero, finite, continuous, differentiable and will be determined later (e.g., $\mu \sim \sigma$ ).

${ }^{10}$ Formula (9) is derived in the usual diagrammatic approach (for $\tilde{V}$ ) and is valid at one loop (even in nonrenormalizable cases, if no higher derivative operators exist and kinetic terms are canonical). Beyond one loop more vacuum "bubble" diagrams exist and then formula (9) receives corrections [29].
} 
The sum is over the eigenvalues ${ }^{11} \tilde{M}_{s}^{2}$ of the matrix $\left(\tilde{M}^{2}\right)_{\alpha \beta}$. Up to $\mathcal{O}\left[(4-d)^{2}\right]$ terms

$$
\begin{aligned}
\left(\tilde{M}^{2}\right)_{\alpha \beta} & =\mu^{4-d}\left[\left(M^{2}\right)_{\alpha \beta}+(4-d) \mu^{-2} N_{\alpha \beta}\right], \\
\alpha, \beta & =\{\phi, \sigma\}
\end{aligned}
$$

where

$$
\begin{aligned}
\left(M^{2}\right)_{\alpha \beta} & =V_{\alpha \beta}, \\
N_{\alpha \beta} & \equiv \mu\left(\mu_{\alpha} V_{\beta}+\mu_{\beta} V_{\alpha}\right)+\left(\mu \mu_{\alpha \beta}-\mu_{\alpha} \mu_{\beta}\right) V,
\end{aligned}
$$

and $\quad \mu_{\alpha}=\partial \mu / \partial \alpha, \quad \mu_{\alpha \beta}=\partial^{2} \mu / \partial \alpha \partial \beta, \quad V_{\alpha}=\partial V / \partial \alpha$, $V_{\alpha \beta}=\partial^{2} V / \partial \alpha \partial \beta$, are nonzero field-dependent quantities. From the last two equations one finds, up to $\mathcal{O}\left[(4-d)^{2}\right]$ terms,

$$
\sum_{s=\phi, \sigma} \tilde{M}_{s}^{4}=\mu^{2(4-d)}\left[\operatorname{Tr} M^{4}+2(4-d) \mu^{-2} \operatorname{Tr}\left(M^{2} N\right)\right] .
$$

Then

$$
\begin{aligned}
U= & \mu(\phi, \sigma)^{4-d}\left\{V-\frac{1}{64 \pi^{2}}\left[\sum_{s=\phi, \sigma} M_{s}^{4}\left(\frac{2}{4-d}-\ln \frac{M_{s}^{2}}{\kappa \mu^{2}(\phi, \sigma)}\right)\right.\right. \\
& \left.\left.+\frac{4 \operatorname{Tr}\left(M^{2} N\right)}{\mu^{2}(\phi, \sigma)}\right]\right\} .
\end{aligned}
$$

The last term is due to the field dependence of $\mu$ and its origin is in the second evanescent term in the rhs of Eq. (12) which cancels the pole to give a finite contribution. We adopt the usual $\overline{\mathrm{MS}}$ scheme here, in which case the counterterms are ${ }^{12}$

$$
\delta U_{\mathrm{ct}}=\frac{\mu(\phi, \sigma)^{4-d}}{64 \pi^{2}} \sum_{s=\phi, \sigma} M_{s}^{4}\left(\frac{2}{4-d}+\ln \kappa-\frac{3}{2}\right),
$$

where $\sum_{s=\phi, \sigma} M_{s}^{4}=V_{\phi \phi}^{2}+V_{\sigma \sigma}^{2}+2 V_{\phi \sigma}^{2}$. Using

\footnotetext{
${ }^{11}$ For any values of the fields, $\operatorname{det}\left(M^{2}\right)_{\alpha \beta}$ is positive provided that $\lambda_{m}^{2} \in\left[3 \lambda_{\phi} \lambda_{\sigma}(3-2 \sqrt{2}), 3 \lambda_{\phi} \lambda_{\sigma}(3+2 \sqrt{2})\right]$ and that $\lambda_{\phi}, \lambda_{\sigma}, \lambda_{m}$ have all the same sign. The eigenvalues are positive if $\lambda_{\phi}, \lambda_{\sigma}, \lambda_{m}$ are positive. If $\lambda_{m}<0, \lambda_{\phi}, \lambda_{\sigma}>0$ one eigenvalue is negative. For $\lambda_{m}^{2}$ outside this interval, restrictions apply to the ratio $\phi^{2} / \sigma^{2}$ for which the eigenvalues are both positive. Note that even in the Standard Model (SM), the Goldstone mode (negative) field-dependent squared mass leads to complex and infrared divergent corrections and then only the real part of the potential is included. A resummation of higher orders in $V$ fixes this wellknown problem [30,31] (see also [16,32]). Here we proceed in general and do not study this issue that affects the ColemanWeinberg term only, but refer the reader to [30,31].

${ }^{12}$ One can use other subtraction schemes, e.g., $\delta U_{c . t .}=$ $\mu^{4-d}\left[a_{1} \phi^{4}\left(1 / \tilde{\epsilon}+c_{1}\right)+a_{2} \phi^{2} \sigma^{2}\left(1 / \tilde{\epsilon}+c_{2}\right)+a_{3} \sigma^{4}\left(1 / \tilde{\epsilon}+c_{3}\right)\right]$ where we denoted $1 / \tilde{\epsilon} \equiv 2 /(4-d)+\ln \kappa-3 / 2$. The case of $\overline{\mathrm{MS}}$ corresponds to $c_{1}=c_{2}=c_{3}=0$.
}

$$
\begin{aligned}
\delta U_{c . t .} \equiv & \mu^{4-d}\left[1 / 4\left(Z_{\lambda_{\phi}}-1\right) \lambda_{\phi} \phi^{4}+1 / 2\left(Z_{\lambda_{m}}-1\right) \lambda_{m} \phi^{2} \sigma^{2}\right. \\
& \left.+1 / 4\left(\lambda_{\sigma}-1\right) \lambda_{\sigma} \sigma^{4}\right]
\end{aligned}
$$

one finds the renormalization coefficients

$$
\begin{aligned}
& Z_{\lambda_{\phi}}=1+\frac{1}{8 \pi^{2}(4-d)}\left(9 \lambda_{\phi}+\lambda_{m}^{2} / \lambda_{\phi}\right) \\
& Z_{\lambda_{m}}=1+\frac{1}{8 \pi^{2}(4-d)}\left(3 \lambda_{\phi}+3 \lambda_{\sigma}+4 \lambda_{m}\right) \\
& Z_{\lambda_{\sigma}}=1+\frac{1}{8 \pi^{2}(4-d)}\left(9 \lambda_{\sigma}+\lambda_{m}^{2} / \lambda_{\sigma}\right) .
\end{aligned}
$$

These Z's have expressions identical to those obtained at one loop with $\mu$ a constant.

After adding the counterterms $\delta U_{c . t}$ we can safely take the limit $d \rightarrow 4$ in the remaining terms $(\mu \neq 0)$, so the renormalized one-loop potential is

$$
\begin{aligned}
U(\phi, \sigma)= & V(\phi, \sigma)+\frac{1}{64 \pi^{2}}\left\{\sum_{s=\phi, \sigma} M_{s}^{4}(\phi, \sigma)\left(\ln \frac{M_{s}^{2}(\phi, \sigma)}{\mu^{2}(\phi, \sigma)}-\frac{3}{2}\right)\right. \\
& +\Delta U(\phi, \sigma)\} \\
\Delta U= & \frac{-4}{\mu^{2}}\left\{V \left[\left(\mu \mu_{\phi \phi}-\mu_{\phi}^{2}\right) V_{\phi \phi}+2\left(\mu \mu_{\phi \sigma}-\mu_{\phi} \mu_{\sigma}\right) V_{\phi \sigma}\right.\right. \\
& \left.+\left(\mu \mu_{\sigma \sigma}-\mu_{\sigma}^{2}\right) V_{\sigma \sigma}\right]+2 \mu\left(\mu_{\phi} V_{\phi \phi}+\mu_{\sigma} V_{\phi \sigma}\right) V_{\phi} \\
& \left.+2 \mu\left(\mu_{\phi} V_{\phi \sigma}+\mu_{\sigma} V_{\sigma \sigma}\right) V_{\sigma}\right\} .
\end{aligned}
$$

In the above $M_{s}^{2}(s=\phi, \sigma)$ are the eigenvalues of the matrix $V_{\alpha \beta}$ given by the roots of equation $\rho^{2}-\rho\left(V_{\phi \phi}+V_{\sigma \sigma}\right)+$ $\left(V_{\phi \phi} V_{\sigma \sigma}-V_{\phi \sigma}^{2}\right)=0 .{ }^{13}$

Equation (19) is a scale-invariant one-loop result. It is a modified version of the Coleman-Weinberg potential (recovered if $\mu$ is a constant) and contains an additional correction $(\Delta U)$. Note that $\Delta U$ is not exactly a counterterm but a finite one-loop effect induced by scale invariance. It is generated when the evanescent coefficient $(4-d)$ in the field-dependent masses of Eq. (12) multiplies the pole $1 /(4-d)$ of the one-loop integral. ${ }^{14}$ This effect is missed in

\footnotetext{
${ }^{13}$ For the particular $V$ of Eq. (3), the eigenvalues $M_{s}(s=\phi, \sigma)$ of $\left(M^{2}\right)_{\alpha \beta}$ are

$$
\begin{aligned}
M_{s}^{2}= & (1 / 2)[\nu \pm \sqrt{\Delta}], \\
\nu \equiv & \left(3 \lambda_{\phi}+\lambda_{m}\right) \phi^{2}+\left(3 \lambda_{\sigma}+\lambda_{m}\right) \sigma^{2}, \\
\Delta= & \left(3 \lambda_{\phi}-\lambda_{m}\right)^{2} \phi^{4}+\left(3 \lambda_{\sigma}-\lambda_{m}\right)^{2} \sigma^{4} \\
& +2 \phi^{2} \sigma^{2}\left[3 \lambda_{m}\left(\lambda_{\phi}+\lambda_{\sigma}\right)-9 \lambda_{\phi} \lambda_{\sigma}+7 \lambda_{m}^{2}\right] .
\end{aligned}
$$

${ }^{14}$ In higher orders, an $n$-loop pole $1 /(4-d)^{n}$, upon multiplication by the $4-d$ coefficient, will actually generate a $1 /(4-d)^{n-1}$ pole, i.e., what we consider usually to account for $n-1$ loop effects. Thus, the order of the singularity is not identical to the loop order in this case.
} 
calculations that are not scale invariant such as the usual DR scheme. Note also that $\Delta U$ vanishes on the tree-level ground state. ${ }^{15}$

$\Delta U$ contains nonpolynomial operators. Even in the minimal case of taking $\mu \sim \sigma$, then the terms in $\Delta U$ proportional to $V V_{\sigma \sigma}$ contain a $\phi^{6} / \sigma^{2}$ term. Similar effective operators are expected to be generated in higher orders. Further, one can Taylor expand the expression of the potential about the ground state, using $\sigma=\langle\sigma\rangle+\delta \sigma$, with $\delta \sigma$ a quantum fluctuation. When doing so, the operator $\phi^{6} / \sigma^{2}$ becomes a series of effective (polynomial) operators

$$
\frac{\phi^{6}}{\sigma^{2}}=\frac{\phi^{6}}{\langle\sigma\rangle^{2}}\left(1-\frac{2 \delta \sigma}{\langle\sigma\rangle}+\frac{3 \delta \sigma^{2}}{\langle\sigma\rangle^{2}}+\cdots\right)
$$

To proceed further, one needs the general expression of the function $\mu=\mu(\phi, \sigma)$. Let us first take $\mu=\mu(\sigma)$ only, which will be justified in the next section; in this case the only possibility is

$$
\mu(\sigma)=z \sigma
$$

which, as a "DR scale," requires $\langle\sigma\rangle \neq 0,\langle\sigma\rangle<\infty$. To be exact, we actually take $\mu(\sigma)=z \sigma^{2 /(d-2)}$ [5], which accounts for the mass dimension of the field $\sigma$. For the one-loop case only (as here) it is safe to use at this stage its limit for $d \rightarrow 4$, so $\mu=z \sigma$. Here $z$ is an arbitrary dimensionless parameter and the dependence of $U$ on $z$ is equivalent to the familiar subtraction scale dependence of $U$ in the usual regularization. With Eq. (22), one obtains the following form of $\Delta U$, which is independent of $z$ :

$$
\Delta U=-\frac{4}{\sigma^{2}}\left[V_{\sigma \sigma}\left(2 \sigma V_{\sigma}-V\right)+2 \sigma V_{\phi} V_{\phi \sigma}\right]
$$

and only the Coleman-Weinberg term depends on $z$. With $V$ of Eq. (3)

$$
\begin{aligned}
\Delta U= & \frac{\lambda_{\phi} \lambda_{m} \phi^{6}}{\sigma^{2}}-\left(16 \lambda_{\phi} \lambda_{m}+6 \lambda_{m}^{2}-3 \lambda_{\phi} \lambda_{\sigma}\right) \phi^{4} \\
& -\left(16 \lambda_{m}+25 \lambda_{\sigma}\right) \lambda_{m} \phi^{2} \sigma^{2}-21 \lambda_{\sigma}^{2} \sigma^{4} .
\end{aligned}
$$

As anticipated, notice the presence of the nonpolynomial operator $\sim \phi^{6} / \sigma^{2}$. This operator is suppressed at large $\langle\sigma\rangle$ or for small mixing $\left(\lambda_{m}\right)$ between $\phi$ and the dilaton $\sigma$. The sign of this operator is controlled by $\lambda_{m}$, assuming $\lambda_{\phi}>0$. When $\lambda_{m}<0$, the term $\lambda_{m} \phi^{6}$ destabilizes the potential for large values of $\phi$. A tuning $\left|\lambda_{m}\right| \ll \lambda_{\phi}$ can compensate to render this term of similar size to $\phi^{4}$ terms; also higher loop orders can generate similar effective operators that may stabilize the potential globally.

\footnotetext{
${ }^{15}$ In a scale-invariant theory $\tilde{V}\left(\alpha z_{i}\right)=\alpha^{d} \tilde{V}\left(z_{i}\right)$; after differentiating with respect to $\alpha$ and setting $\alpha \rightarrow 1$ then $d \times \tilde{V}=\sum_{i} z_{i} \partial \tilde{V} / \partial z_{i}, z_{i}: \phi, \sigma$. From min conditions with respect to $\phi, \sigma: \tilde{V}_{\phi}=\tilde{V}_{\sigma}=0 \Rightarrow V_{\min }=0, \Delta U_{\min }=0(d \rightarrow 4)$.
}

For the special case of a nontrivial classical vacuum of Eq. (5), when $\lambda_{m}^{2}=\lambda_{\phi} \lambda_{\sigma}$, Eq. (24) becomes

$$
\begin{aligned}
\Delta U= & \frac{\lambda_{m}}{\lambda_{\phi}^{2} \sigma^{2}}\left(\lambda_{\phi} \phi^{2}+\lambda_{m} \sigma^{2}\right) \\
& \times\left[\lambda_{\phi}^{2} \phi^{4}-4 \lambda_{\phi}\left(4 \lambda_{\phi}+\lambda_{m}\right) \phi^{2} \sigma^{2}-21 \lambda_{m}^{2} \sigma^{4}\right] .
\end{aligned}
$$

This expression vanishes on the tree-level ground state, ${ }^{16}$ when $\lambda_{\phi}\langle\phi\rangle^{2}+\lambda_{m}\langle\sigma\rangle^{2}=0\left(\lambda_{m}<0, \lambda_{\phi}>0\right)$.

In conclusion, the expression of $U$ at one loop is manifestly scale invariant

$$
\begin{aligned}
U(\phi, \sigma)= & V(\phi, \sigma)+\frac{1}{64 \pi^{2}}\left[\sum_{s=\phi, \sigma} M_{s}^{4}(\phi, \sigma)\left(\ln \frac{M_{s}^{2}(\phi, \sigma)}{z^{2} \sigma^{2}}-\frac{3}{2}\right)\right. \\
& +\Delta U(\phi, \sigma)]
\end{aligned}
$$

with $\Delta U$ as in Eqs. (24) or (25) and $V$ of Eq. (3) and where the "standard" Coleman-Weinberg term is modified into a scale-invariant form. This is the main result of this section, valid under our assumption $\mu(\sigma)=z \sigma$. Further, $U$ can be Taylor expanded about $\langle\sigma\rangle$. With no mass scale in the theory, from minimizing $U$ one can only predict ratios of vevs, so all masses are generated by $\langle\sigma\rangle$ after spontaneous breaking of scale symmetry.

\section{MORE GENERAL $\mu(\phi, \sigma)$ AND IMPLICATIONS}

The subtraction function could, in principle, be more general and could depend on $\phi$ too, $\mu=\mu(\phi, \sigma)$. In this section we show that such dependence is not physical and conclude that $\mu$ must be a function on the dilaton only. First, consider the following example:

$$
\mu(\phi, \sigma)=z\left(\xi_{\phi} \phi^{2}+\xi_{\sigma} \sigma^{2}\right)^{1 / 2} .
$$

This was used in earlier similar studies $[2,4]$ where scaleinvariant models had a nonminimal coupling to gravity, with this expression to fix the Planck scale upon spontaneous breaking of scale symmetry. ${ }^{17}$ With this $\mu$ and $V$ of Eq. (3) one finds that $\Delta U$ contains leading power terms $\phi^{8}$ and $\sigma^{8}$ as shown in

\footnotetext{
${ }^{16}$ At the loop level the ground state is changed slightly, but we ignore that effect here.

${ }^{17}$ The nonminimal coupling is $\mathcal{L}_{G}=-\frac{1}{2}\left(\xi_{\phi} \phi^{2}+\xi_{\sigma} \sigma^{2}\right) R$, and is added in some models to generate the Planck mass from $\mu(\sigma, \phi \ldots)$ in (spontaneously broken) scale-invariant theories $[2,4]$. The relative signs of $\xi_{\phi}, \xi_{\sigma}$ are important to ensure a positive Newton constant (for a review see [33]). When going to the Einstein frame, this coupling generates a suppression of the treelevel potential by a factor $1 /\left(\xi_{\phi} \phi^{2}+\xi_{\sigma} \sigma^{2}\right)^{2}$, while in the case discussed in the text (where no such coupling is included), such suppression is shown to be generated in $\Delta U$ at one loop; see later.
} 


$$
\begin{aligned}
\Delta U= & -\left(\xi_{\phi} \phi^{2}+\xi_{\sigma} \sigma^{2}\right)^{-2}\left[\left(21 \lambda_{\phi} \xi_{\phi}+\lambda_{m} \xi_{\sigma}\right) \xi_{\phi} \lambda_{\phi} \phi^{8}\right. \\
& \left.+\left(21 \lambda_{\sigma} \xi_{\sigma}+\lambda_{m} \xi_{\phi}\right) \xi_{\sigma} \lambda_{\sigma} \sigma^{8}+\cdots\right] .
\end{aligned}
$$

The dots stand for the remaining $\phi^{6} \sigma^{2}, \phi^{4} \sigma^{4}$, and $\phi^{2} \sigma^{6}$ terms, which we do not display since their coefficients are too long. The coefficients of $\phi^{8}, \sigma^{8}$ are positive irrespective of the values of $\xi_{\phi, \sigma}$, if $\lambda_{m}^{2} \geq 21^{2} \lambda_{\phi} \lambda_{\sigma}$, (with $\lambda_{\phi, \sigma}>0$ ). This condition is not respected on the ground state of $V$ (with $\lambda_{m}^{2}=\lambda_{\phi} \lambda_{\sigma}$ ). We thus encounter terms unbounded from below, that otherwise vanish on the tree-level ground state. A small fluctuation about the critical point can then destabilize the potential.

It is intriguing that even if the classical $V$ contains no interaction terms between "visible" $(\phi)$ and "hidden" $(\sigma)$ sectors, i.e., $\lambda_{m}=0$, such terms are still generated by quantum corrections, for $\mu(\phi, \sigma)$ of Eq. (27). Indeed, one has

$$
\begin{aligned}
\left.\Delta U\right|_{\lambda_{m}=0}= & -3\left[\xi _ { \phi } \xi _ { \sigma } \left[\lambda_{\phi}\left(9 \lambda_{\phi}+\lambda_{\sigma}\right) \phi^{6} \sigma^{2}\right.\right. \\
& \left.+\lambda_{\sigma}\left(\lambda_{\phi}+9 \lambda_{\sigma}\right) \phi^{2} \sigma^{6}\right]+7\left(\lambda_{\phi}^{2} \xi_{\phi}^{2} \phi^{8}+\lambda_{\sigma}^{2} \xi_{\sigma}^{2} \sigma^{8}\right) \\
& \left.-\left(\xi_{\phi}^{2}+\xi_{\sigma}^{2}\right) \lambda_{\phi} \lambda_{\sigma} \phi^{4} \sigma^{4}\right]\left(\xi_{\phi} \phi^{2}+\xi_{\sigma} \sigma^{2}\right)^{-2} .
\end{aligned}
$$

This simplifies further if also $\lambda_{\sigma}=\lambda_{m}^{2} / \lambda_{\phi} \rightarrow 0$, but the term $\propto \xi_{\phi} \xi_{\sigma} \lambda_{\phi}^{2} \phi^{6} \sigma^{2}$ does not vanish. Such terms ultimately arise from the expression of the $\mu$-dependent factor in $\tilde{V}$, via terms like $\mu_{\phi} V_{\phi} V_{\phi \phi}$ and $\left(\mu \mu_{\phi \phi}-\mu_{\phi}^{2}\right) V_{\phi \phi}$ in Eq. (19). The two sectors still "communicate" at the quantum level, due to scale invariance even if they are classically decoupled. This concerning effect is only removed for vanishing $\xi_{\phi}$ or $\xi_{\sigma}$, which means $\mu \propto \sigma{ }^{18}$

More generally, consider

$$
\mu(\phi, \sigma)=z \sigma e^{g(\phi / \sigma)} .
$$

Here, $g$ is some arbitrary function of the ratio $\phi / \sigma$. In this case, in the classical "decoupling" limit $\lambda_{m} \rightarrow 0$ also with $\lambda_{\sigma}=\lambda_{m}^{2} / \lambda_{\phi} \rightarrow 0$, there are nonvanishing quantum interactions terms:

$$
\left.\Delta U\right|_{\lambda_{m}=0}=-3 \lambda_{\phi}^{2}\left[8 \frac{\phi^{5}}{\sigma} g^{\prime}(\phi / \sigma)+\frac{\phi^{6}}{\sigma^{2}} g^{\prime \prime}(\phi / \sigma)\right] .
$$

Again, the two sectors still communicate at the quantum level only. To avoid such concerning behavior, we must take $g=0$ or a constant. ${ }^{19}$ Therefore, the subtraction function is independent of $\phi$ and thus $\mu(\sigma)=z \sigma$. This result is the minimal scenario used in the previous section and justifies our choice in Eq. (22) and our result in

\footnotetext{
${ }^{18}$ up to a relabeling, see the symmetry $\phi \leftrightarrow \sigma$, at which stage one decides which field denotes the dilaton.

${ }^{19}$ We disregard a second solution for which the rhs of Eq. (31) vanishes, since it is not continuous at $\phi=0$.
}

Eq. (26). We conclude that it is the dilaton alone that generates the subtraction scale after spontaneous breaking of scale symmetry.

\section{THE MASS SPECTRUM}

Let us minimize the one-loop potential $U$. We restrict the analysis to the simpler case of a hierarchy of the couplings considered in $[2,15]$. We take

$$
\lambda_{\sigma} \ll\left|\lambda_{m}\right| \ll \lambda_{\phi}
$$

To enforce this hierarchy, introduce $\lambda_{m}=\tilde{\lambda}_{m} \varepsilon$ and $\lambda_{\sigma}=\tilde{\lambda}_{\sigma} \varepsilon^{2}$, where $\varepsilon \sim 1 / M_{\text {Planck }}^{2} \ll 1$, and $\lambda_{\phi}, \tilde{\lambda}_{m}$, and $\tilde{\lambda}_{\sigma}$ are now of similar magnitude. One then expands $U$ up to $\mathcal{O}\left(\varepsilon^{3}\right) \sim \mathcal{O}\left(\lambda_{m}^{3}\right)$,

$$
\begin{aligned}
U= & \frac{\lambda_{\phi}}{4} \phi^{4}+\frac{\lambda_{m}}{2} \phi^{2} \sigma^{2}+\frac{\lambda_{\sigma}}{4} \sigma^{4}+\frac{1}{64 \pi^{2}}\left\{M_{1}^{4}\left[\ln \frac{M_{1}^{2}}{z^{2} \sigma^{2}}-\frac{3}{2}\right]\right. \\
& +M_{2}^{4}\left[\ln \frac{M_{2}^{2}}{z^{2} \sigma^{2}}-\frac{3}{2}\right]+\lambda_{\phi} \lambda_{m} \frac{\phi^{6}}{\sigma^{2}} \\
& \left.-\left(16 \lambda_{\phi} \lambda_{m}+6 \lambda_{m}^{2}-3 \lambda_{\phi} \lambda_{\sigma}\right) \phi^{4}-16 \lambda_{m}^{2} \phi^{2} \sigma^{2}\right\}+\mathcal{O}\left(\lambda_{m}^{3}\right) .
\end{aligned}
$$

One can minimize $U$ and find the solution for $\langle\phi\rangle /\langle\sigma\rangle$ that satisfies $U_{\phi}=U_{\sigma}=0 ; U$ being manifestly scale invariant, these conditions ensure a flat direction exists and also that vacuum energy vanishes in this order. One finds

$\frac{\langle\phi\rangle^{2}}{\langle\sigma\rangle^{2}}=-\frac{\lambda_{m}}{\lambda_{\phi}}\left[1-\frac{6 \lambda_{\phi}}{64 \pi^{2}}\left(4 \ln 3 \lambda_{\phi}-17 / 3\right)\right]+\mathcal{O}\left(\lambda_{m}^{2}\right)$.

This brings a correction to the tree-level case, Eq. (5); here $\lambda_{m}<0, \lambda_{\phi}>0$ and $\lambda_{m}^{2}=\lambda_{\phi} \lambda_{\sigma}$. To obtain Eq. (34) we fixed the subtraction parameter $z$ under the $\log$ term in $U$ to

$$
z=\langle\phi\rangle /\langle\sigma\rangle, \quad \text { then } \mu \rightarrow\langle\phi\rangle
$$

on the ground state. This value for $\mu$ is the standard choice for the subtraction scale, made to minimize the ColemanWeinberg log-term dependence on it. As mentioned, $\Delta U$ itself is scheme independent (being independent of $z$ ).

The potential in (33) is scale invariant; the dilaton remains massless at one loop while the Higgs-like scalar $\phi$ has a mass

$$
m_{\tilde{\phi}}^{2}=\left[U_{\phi \phi}+U_{\sigma \sigma}\right]_{\min } .
$$

Let us consider only the contribution $\delta m_{\tilde{\phi}}^{2}$ from $\Delta U$ alone to the mass of $\phi$. The interest is to examine if potentially "dangerous" corrections of the type $\lambda_{\phi}^{2}\langle\sigma\rangle^{2}$, etc. can emerge from the new contribution $\Delta U$. These would require an additional tuning (of $\lambda_{\phi}$ ) beyond that of $\lambda_{m}$ done at the tree 
level, in order to keep $\phi$ light compared to $\langle\sigma\rangle \sim M_{\text {Planck }}$. In general, one has

$$
\begin{aligned}
\delta m_{\tilde{\phi}}^{2}= & \frac{1}{64 \pi^{2}}\left(\Delta U_{\phi \phi}+\Delta U_{\sigma \sigma}\right)_{\min } \\
= & \frac{-\langle\sigma\rangle^{2}}{32 \pi^{2}}\left[4 \lambda_{m}^{2}(4+13 \rho)+18 \lambda_{\sigma}\left(7 \lambda_{\sigma}-\lambda_{\phi} \rho\right)\right. \\
& \left.+\lambda_{m}\left[25 \lambda_{\sigma}(1+\rho)-3 \lambda_{\phi} \rho\left(-32+5 \rho+\rho^{2}\right)\right]\right]
\end{aligned}
$$

where $\rho=\langle\phi\rangle^{2} /\langle\sigma\rangle^{2}$. This mass correction contains terms proportional to $\lambda_{m}$ or $\lambda_{\sigma}=\lambda_{m}^{2} / \lambda_{\phi} \ll \lambda_{m}$ but not to $\lambda_{\phi}$ alone. Therefore no extra tuning is needed beyond that at the classical level of Eqs. (5) and (6), in order to maintain $\delta m_{\tilde{\phi}}^{2}$ and $m_{\tilde{\phi}}^{2} \sim\langle\phi\rangle^{2} \sim \lambda_{m}\langle\sigma\rangle^{2}$ close to the electroweak scale. It is possible that this nice behavior survives to higher or all orders, as a result of the manifest scale invariance and spontaneous breaking of this symmetry. This suggests that the hierarchy problem could be solved with only one initial (classical) tuning of $\lambda_{m}$ (no tuning of $\lambda_{\phi}$ ).

\section{FURTHER REMARKS}

The method we used to generate dynamically the subtraction scale of the DR scheme, as the dilaton vev, deserves further study.

First, note that the potential $U$ must respect the CallanSymanzik equation, i.e., it must be independent of the choice of the dimensionless parameter $z$ and thus of the subtraction scale $z\langle\sigma\rangle$ after spontaneous scale symmetry breaking [5]. In our one-loop approximation this demands that

$$
\frac{d U}{d \ln z}=\left(\frac{\partial U}{\partial \ln z}+\beta_{\lambda_{j}} \frac{\partial}{\partial \lambda_{j}}\right) U=O\left(\lambda^{3}\right)
$$

where $U$ is that of Eq. (26) and $\Delta U$ of (24) and the Coleman-Weinberg term is the only one that depends explicitly on $z$. To check if condition (38) is respected, we need the one-loop beta functions of the theory; these are obtained from the condition that the "bare" couplings of the Lagrangian are independent of subtraction scale $z\langle\sigma\rangle$, where $z$ is arbitrary: $d\left(\lambda_{j} Z_{\lambda_{j}}\right) / d \ln z=0$, where $j=$ $\phi, m, \sigma$ (fixed) and $Z_{\lambda_{j}}$ are given in Eq. (17). ${ }^{20}$ One finds

\footnotetext{
${ }^{20}$ To be exact, in $d=4-2 \epsilon$, one actually imposes $d\left((z \sigma)^{2 \epsilon} \lambda_{j} Z_{\lambda_{j}}\right) / d \ln z=0$, giving that beta functions are shifted from those above, $\beta_{\lambda_{j}}=-2 \epsilon \lambda_{j}+(\ldots)$ where (...) denotes the right-hand side in each of the equations in (39).
}

$$
\begin{aligned}
& \beta_{\lambda_{\phi}}=\frac{d \lambda_{\phi}}{d \ln z}=\frac{1}{8 \pi^{2}}\left(9 \lambda_{\phi}^{2}+\lambda_{m}^{2}\right) \\
& \beta_{\lambda_{m}}=\frac{d \lambda_{m}}{d \ln z}=\frac{1}{8 \pi^{2}}\left(3 \lambda_{\phi}+4 \lambda_{m}+3 \lambda_{\sigma}\right) \lambda_{m} \\
& \beta_{\lambda_{\sigma}}=\frac{d \lambda_{\sigma}}{d \ln z}=\frac{1}{8 \pi^{2}}\left(\lambda_{m}^{2}+9 \lambda_{\sigma}^{2}\right)
\end{aligned}
$$

which are the same as in the case where the theory was regularized with $\mu=$ constant. ${ }^{21}$ Using these beta functions one easily checks that Eq. (38) is respected. This shows that the change of parameter $z$ is "moved" into the running couplings $\mathrm{s}^{22}$ of the potential and physics is indeed independent of $z: U\left(\lambda_{j}(z), z\right)=U\left(\lambda_{j}\left(z_{0}\right), z_{0}\right)$, where $j=\phi, m, \sigma$ and $z, z_{0}$ are different subtraction parameters (ultimately corresponding to subtraction scales $\left.z\langle\sigma\rangle, z_{0}\langle\sigma\rangle\right)$.

Regarding the renormalizability of scale-invariant models, previous studies [23] identified at three-loop order a UV counterterm to the original Lagrangian $L$, of the form

$$
\frac{1}{\left(16 \pi^{2}\right)^{3}} \frac{1}{(4-d)^{2}}\left(\frac{\xi_{\phi}}{\xi_{\sigma} \sigma^{2}}\right)^{2}\left(\square \phi^{2}\right)^{2}
$$

In [23] $\mu \sim\left(\xi_{\phi} \phi^{2}+\xi_{\sigma} \sigma^{2}\right)^{1 / 2}$, just like in Eq. (27). This UV divergence was due to a new vertex generated by the Taylor expansion of $\mu(\phi, \sigma)$ with respect to $\phi$; this vertex is ultimately due to new interactions that $\mu(\phi, \sigma)$ itself brought in $\tilde{V}$ but is absent in initial $V$. Given this counterterm, the theory is then nonrenormalizable and nonlocal. The same conclusion is expected for any subtraction function that depends on additional fields other than dilaton.

However, we showed that $\mu=z \sigma$ (Sec. IV), so the above three-loop counterterm is absent because we have $\xi_{\phi}=0$. Despite this, the standard expectation is that higher loop orders still generate higher dimensional counterterms and the theory is nonrenormalizable, due to the presence in $U$ of the nonpolynomial term $\phi^{6} / \sigma^{2}$. [One can still explore the possibility that in a scale symmetry-preserving calculation, all poles in quantum corrected $L$ be those that renormalize its initial couplings and fields only (i.e., renormalizability), without other UV counterterms. This problem deserves careful investigation and is beyond the goal of this paper.]

As a result of a manifestly scale-invariant regularization, the (mass) $)^{2}$ of $\phi$ contains quadratic contributions $\lambda_{m}\langle\sigma\rangle^{2}$ and corrections suppressed by $1 /\langle\sigma\rangle^{2}$, in addition to loglike terms $\ln \langle\sigma\rangle \sim \ln \mu$ present in the usual DR scheme. Our method to generate the subtraction scale via spontaneous breaking in a dilaton-modified DR can also be implemented in other regularizations. Also note that the role of $\mu \sim \sigma$ as a

\footnotetext{
${ }^{21}$ This is expected since we only found new finite terms, but no new counterterms.

${ }^{22}$ Thus, there is no dilatation anomaly, yet the couplings still run as usual $[5,6]$.
} 
finite, nonzero DR scale means that only nonzero, finite $\langle\sigma\rangle$ is allowed. In fixing its actual numerical value, Planck scale physics (gravity) is expected to play a role.

Although we do not explore them here, our results can have interesting applications to phenomenology, such as model building beyond SM [13-15]. For reference only, we provide below the one-loop potential in the scale-invariant version of the $\mathrm{SM}^{23}$ extended by the dilaton. With the usual $\mathrm{CW}$ part $\delta U_{\mathrm{CW}},{ }^{24}$ the one-loop scalar potential in the SM is [with $M_{\phi}^{2}, M_{\sigma}^{2}$ as in Eq. (20)]

$$
\begin{aligned}
U= & \frac{\lambda_{\phi}}{4} \phi^{4}+\frac{\lambda_{m}}{2} \phi^{2} \sigma^{2}+\frac{\lambda_{\sigma}}{4} \sigma^{4}+\delta U_{\mathrm{CW}}+\frac{1}{64 \pi^{2}}\left\{\lambda_{\phi} \lambda_{m} \frac{\phi^{6}}{\sigma^{2}}-\left(16 \lambda_{\phi} \lambda_{m}+6 \lambda_{m}^{2}-3 \lambda_{\phi} \lambda_{\sigma}\right) \phi^{4}-\left(16 \lambda_{m}+25 \lambda_{\sigma}\right) \lambda_{m} \phi^{2} \sigma^{2}-21 \lambda_{\sigma}^{2} \sigma^{4}\right. \\
& \left.+M_{\sigma}^{4} \ln \frac{M_{\sigma}^{2}}{z^{2} \sigma^{2}}-\frac{3}{2}\left[\left(9 \lambda_{\phi}^{2}+\lambda_{m}^{2}\right) \phi^{4}+2 \lambda_{m}\left(3 \lambda_{\phi}+4 \lambda_{m}+3 \lambda_{\sigma}\right) \phi^{2} \sigma^{2}+\left(\lambda_{m}^{2}+9 \lambda_{\sigma}^{2}\right) \sigma^{4}\right]\right\} .
\end{aligned}
$$

The last two lines give the new correction $\Delta U$, while in the $\mathrm{CW}$ term one uses $\mu=z \sigma$ with $z=\langle\phi\rangle /\langle\sigma\rangle$. This equation can be used as the starting point in phenomenological studies.

\section{CONCLUSIONS}

Scale-invariant theories are often considered to address the hierarchy problem of the Standard Model. However, the regularization of their quantum corrections breaks explicitly the scale symmetry that one wants to study. This is because all regularizations introduce a dimensionful parameter, e.g., the couplings in DR, the UV scale in other regularizations, etc. One can avoid this problem by using a manifestly scaleinvariant regularization in which the Goldstone mode of this symmetry (dilaton) plays a central role. We used a dilaton-dependent subtraction function $\mu=\mu(\sigma)$ that replaces the ordinary subtraction scale. We applied this procedure to the DR scheme, to obtain a scale-invariant one-loop scalar potential $U(\phi, \sigma)$ for the (Higgs-like) scalar $\phi$ and dilaton $\sigma$. After spontaneous breaking of scale invariance when $\langle\sigma\rangle \neq 0$, all mass scales of the theory, including the usual subtraction scale, are generated from this single vev.

The scale invariance of the action in $d=4-2 \epsilon$ and the usual rescaling $\lambda \rightarrow \mu^{2 \epsilon} \lambda$ that ensures dimensionless quartic couplings change the potential $V(\phi, \sigma) \rightarrow \mu(\sigma)^{2 \epsilon} V(\phi, \sigma)$ which now contains new (evanescent) interactions due to the field dependence of $\mu$. At the quantum level, these interactions generate new, finite corrections.

We found a new (finite) one-loop correction $\Delta U$ to the potential, overlooked by previous studies, that is present beyond the usual Coleman-Weinberg term which is also

\footnotetext{
${ }^{23}$ This is just the SM with no classical mass term for the Higgs in the Lagrangian.

${ }^{24} \delta U_{\mathrm{CW}}=\frac{1}{64 \pi^{2}} \sum_{i} N_{i} M_{i}^{4}\left[\ln M_{i}^{2} / \mu^{2}(\phi, \sigma)-C_{i}\right], \quad i=(G, S, W, Z, t)$ for Goldstone bosons, real scalars, gauge bosons, top, respectively, with $\left(N_{G}, N_{S}, N_{W}, N_{Z}, N_{t}\right)=(3,1,6,3,-12) . C_{i}=3 / 2$ for fermions or scalars and $5 / 6$ for gauge bosons. $M_{G}^{2}=\lambda_{\phi} \phi^{2}+\lambda_{m} \sigma^{2}, M_{W}^{2}=\frac{1}{4} g^{2} \phi^{2}, M_{Z}^{2}=\frac{1}{4}\left(g^{2}+g^{\prime 2}\right) \phi^{2}, M_{t}^{2}=$ $\frac{1}{2} y_{t}^{2} \phi^{2}$. The potential (3) of Higgs dilaton, $V=\lambda_{\phi}|H|^{4}+$ $\lambda_{m}|H|^{2} \sigma^{2}+\left(\lambda_{\sigma} / 4\right) \sigma^{4}$ with $H=(0, \phi) / \sqrt{2}$ (unitary gauge).
}

modified into a scale-invariant form. For the minimal case $\mu(\sigma)=z \sigma$, it was shown that $\Delta U$ also contains a nonpolynomial operator $\propto \phi^{6} / \sigma^{2}$ with a known, finite coupling. After spontaneous breaking of scale invariance, this operator generates a series of (polynomial) terms suppressed by powers of $\langle\sigma\rangle \neq 0$. At higher loop orders, more such operators are expected.

Technically, $\Delta U$ is generated from an evanescent correction $(\alpha \epsilon)$ to the field-dependent masses of the states "running" in the loop correction to the potential, which cancels the pole $(\propto 1 / \epsilon)$ of the momentum integral, to give rise to a finite $\Delta U$. And since it is finite, $\Delta U$ was found to be independent of the subtraction (dimensionless) parameter $(z)$. Of course physics must be independent of $z$ and of the subtraction scale $\mu(\langle\sigma\rangle)=z\langle\sigma\rangle$ after spontaneous breaking of scale symmetry. To check this we showed that the full one-loop potential $U(\phi, \sigma)$ does respect the Callan-Symanzik equation.

Further, the correction from $\Delta U$ to the mass of the Higgslike scalar $\phi$ remains under control (small) without additional tuning beyond that done at the tree level to enforce the hierarchy $\langle\phi\rangle \ll\langle\sigma\rangle$. It is possible that this behavior survives in higher orders, in a manifest scale-invariant calculation. This could provide a solution to the hierarchy problem beyond the one-loop order discussed here.

More general subtraction functions that depend on both $\sigma$ and $\phi$ were ruled out because in this case there are quantum operators that force the visible sector $(\phi)$ and hidden sector $(\sigma)$ to interact in $d=4$ even in their classical decoupling limit $\left(\lambda_{m}=0\right)$. Avoiding this behavior dictates that only the dilaton alone can generate the subtraction scale after spontaneous breaking of scale symmetry. Thus $\mu$ depends on $\sigma$ only, as considered above. The above approach and the scale-invariant regularization are of interest to theories that study scale invariance at the quantum level.

\section{ACKNOWLEDGMENTS}

The author thanks H. M. Lee, E. Dudas and G. G. Ross for discussions on this topic. This work was supported by a grant of the Romanian National Authority for Scientific Research (CNCS-UEFISCDI) under Project No. PN-II-IDPCE-2011-3-0607. 
[1] F. Englert, C. Truffin, and R. Gastmans, Conformal invariance in quantum gravity, Nucl. Phys. B117, 407 (1976); S. Deser, Scale invariance and gravitational coupling, Ann. Phys. (N.Y.) 59, 248 (1970).

[2] M. Shaposhnikov and D. Zenhausern, Quantum scale invariance, cosmological constant and hierarchy problem, Phys. Lett. B 671, 162 (2009).

[3] C. Wetterich, Cosmology and the fate of dilatation symmetry, Nucl. Phys. B302, 668 (1988).

[4] M. Shaposhnikov and D. Zenhausern, Scale invariance, unimodular gravity and dark energy, Phys. Lett. B 671, 187 (2009).

[5] C. Tamarit, Running couplings with a vanishing scale anomaly, J. High Energy Phys. 12 (2013) 098. See also [8].

[6] R. Armillis, A. Monin, and M. Shaposhnikov, Spontaneously broken conformal symmetry: Dealing with the trace anomaly, J. High Energy Phys. 10 (2013) 030.

[7] R. Foot, A. Kobakhidze, K. L. McDonald, and R. R. Volkas, Poincaré protection for a natural electroweak scale, Phys. Rev. D 89, 115018 (2014).

[8] R. Foot, A. Kobakhidze, K. L. McDonald, and R. R. Volkas, A Solution to the hierarchy problem from an almost decoupled hidden sector within a classically scale invariant theory, Phys. Rev. D 77, 035006 (2008).

[9] C. T. Hill, Is the Higgs boson associated with ColemanWeinberg dynamical symmetry breaking?, Phys. Rev. D 89, 073003 (2014).

[10] B. Grinstein and P. Uttayarat, A very light dilaton, J. High Energy Phys. 07 (2011) 038.

[11] W. D. Goldberger, B. Grinstein, and W. Skiba, Distinguishing the Higgs Boson from the Dilaton at the Large Hadron Collider, Phys. Rev. Lett. 100, 111802 (2008).

[12] R. H. Boels and W. Wormsbecher, Spontaneously broken conformal invariance in observables, arXiv:1507.08162.

[13] A. Farzinnia, H. J. He, and J. Ren, Natural Electroweak Symmetry Breaking from Scale Invariant Higgs Mechanism, Phys. Lett. B 727, 141 (2013).

[14] E. Gabrielli, M. Heikinheimo, K. Kannike, A. Racioppi, M. Raidal, and C. Spethmann, Towards completing the standard model: Vacuum stability, EWSB and dark matter, Phys. Rev. D 89, 015017 (2014).

[15] K. Allison, C. T. Hill, and G. G. Ross, Ultra-weak sector, Higgs boson mass, and the dilaton, Phys. Lett. B 738, 191 (2014).

[16] K. Endo and Y. Sumino, A scale-invariant Higgs sector and structure of the vacuum, J. High Energy Phys. 05 (2015) 030 .

[17] S. Iso, N. Okada, and Y. Orikasa, Classically conformal $B^{-}$L extended standard model, Phys. Lett. B 676, 81 (2009).
[18] S. Iso and Y. Orikasa, TeV Scale B-L model with a flat Higgs potential at the Planck scale: In view of the hierarchy problem, Prog. Theor. Exp. Phys. 2013, 023 B08 (2013).

[19] E. J. Chun, S. Jung, and H. M. Lee, Radiative generation of the Higgs potential, Phys. Lett. B 725, 158 (2013); Erratum: Radiative generation of the Higgs potential, Phys. Lett. B 730, 357(E) (2014).

[20] V. V. Khoze, Inflation and dark matter in the Higgs portal of classically scale invariant standard model, J. High Energy Phys. 11 (2013) 215.

[21] V. V. Khoze, C. McCabe, and G. Ro, Higgs vacuum stability from the dark matter portal, J. High Energy Phys. 08 (2014) 026.

[22] S. Abel and A. Mariotti, Novel Higgs potentials from gauge mediation of exact scale breaking, Phys. Rev. D 89, 125018 (2014).

[23] M.E. Shaposhnikov and F. V. Tkachov, Quantum scale-invariant models as effective field theories, arXiv:0905.4857.

[24] F. Gretsch and A. Monin, Dilaton: Saving conformal symmetry, Phys. Rev. D 92, 045036 (2015).

[25] W. A. Bardeen, Reports No. FERMILAB-CONF-95-391-T, No. C95-08-27.3.

[26] S. R. Coleman and E. J. Weinberg, Radiative corrections as the origin of spontaneous symmetry breaking, Phys. Rev. D 7, 1888 (1973).

[27] E. Gildener and S. Weinberg, Symmetry breaking and scalar bosons, Phys. Rev. D 13, 3333 (1976).

[28] A. Kobakhidze, Quantum relaxation of the Higgs mass, Eur. Phys. J. C 75, 384 (2015).

[29] L. J. Boya and J. Casahorran, Two loop effective potential in $\lambda \phi^{4}$ in two-dimensions, Nuovo Cim. A 100, 907 (1988); C. Ford, I. Jack, and D. R. T. Jones, The standard model effective potential at two loops, Nucl. Phys. B387, 373 (1992); Erratum: The standard model effective potential at two loops, Nucl. Phys. B504, 551(E) (1997).

[30] J. Elias-Miro, J. R. Espinosa, and T. Konstandin, Taming infrared divergences in the effective potential, J. High Energy Phys. 08 (2014) 034.

[31] S.P. Martin, Taming the Goldstone contributions to the effective potential, Phys. Rev. D 90, 016013 (2014).

[32] E. J. Weinberg and A. Q. Wu, Understanding complex perturbative effective potentials, Phys. Rev. D 36, 2474 (1987); see also S. G. Matinyan and B. Muller, Quantum fluctuations and dynamical chaos, Phys. Rev. Lett. 78, 2515 (1997); S. G. Matinyan and B. Muller, Quantum fluctuations and dynamical chaos: An Effective potential approach, Found. Phys. 27, 1237 (1997); K. E. Cahill, An effective potential that is real, Phys. Rev. D 52, 4704 (1995).

[33] I. Oda, Conformal Higgs gravity, Adv. Stud. Theor. Phys. 9, 595 (2015). 